\title{
Blood Coagulation Thromboplastine Time Measurements on a Nanoparticle Coated Quartz Crystal Microbalance Biosensor in Excellent Agreement with Standard Clinical Methods
}

\author{
Munawar Hussain ${ }^{1}$, Stefan Sinn ${ }^{1}$, Martin Zeilinger ${ }^{2}$, Hinnak Northoff ${ }^{1}$, Peter A Lieberzeit ${ }^{2}$ and Frank K Gehring ${ }^{1 *}$ \\ ${ }^{1}$ Biosensor Research Group, Institute of Clinical and Experimental Transfusion Medicine, Tuebingen University, Germany \\ ${ }^{2}$ University of Vienna, Department of Analytical Chemistry, Währinger Strasse, Vienna, Austria
}

\begin{abstract}
Measurements of parameters of hemostasis like thromboplastine time (PT) have primary significance in many clinical settings including extensive surgery, dialysis or innate disorders of hemostasis. Recently, several reports have documented the principle suitability of Quartz Crystal Microbalances (QCM) for measuring parameters of hemostasis like PT or platelet aggregation. But for the establishment of an exact QCM based method as alternative to standard coagulometer measuring QCM coatings with significantly enhanced robustness and reusability have to be worked out. For this purpose we utilized a new semi-automated equipment qCell $\mathrm{T}$ for measuring and compared different coatings consisting of polymer films and absorbed nanoparticles (NPs). We demonstrated that affinity based poly ethylene NPs absorbed to polymer films on the QCM constitute a powerful tool with no need for pretreatment for measuring PT in whole blood samples in real time, while these coatings are reusable up to 10 times. PT measurements in excellent agreement with coagulometer tests pave the way for possible future application of QCM in clinical routine.
\end{abstract}

Keywords: QCM sensor; Thromboplastine time; Polymer nanoparticles; qCell T; 3T analytik

\section{Introduction}

The use of NPs in medicine spreads rapidly. NPs play more and more an important role for drug delivery, therapy and diagnostic. In difference to bulk materials NPs bear unique properties as small size, large surface area to mass ratio and high reactivity [1]. Since NPs interact with blood coagulation [2] and NPs can also be imprinted with specific domains (mixed in a pore) [3,4], the NPs bear in principle large potential as basic quartz coating. Therefore we utilized NPs on different polymers for coagulation measurements. Hemostasis monitoring during surgical operations has fundamental significance especially in cardiac surgery involving extracorporal circulation devices. In such procedures blood parameters can drastically change within the lapse of a few seconds to minutes due to exposure of patients' haemostatic system to large disturbances such as haemo-dilution or anticoagulation. Additionally some unwanted interactions of the blood with artificial surfaces may cause coagulation activation, blood platelet aggregation [5] or proinflammatory effects [6,7]. In such conditions frequent monitoring of the patients' haemostasis status is often crucial for suitable therapeutic directions and decisions $[8,9]$. Regarding this purpose a large number of instruments with "point of care" capability have emerged. These devices were developed for user friendly purposes such as preparation without complex procedures during surgical intervention. PT measurements using QCM transducers as biosensors $[10,11]$ have a great potential in such sorts of relevant applications $[3,12,13]$. In this regard, recently a suitable instrument qCell T has been launched by $3 \mathrm{~T}$ analytik, Tuttlingen, Germany. By measuring resonance frequency as well as dissipation, the instrument itself has a wide range of applications. Mass changes and properties as density and viscosity of attached layers and wetting liquids can properly be measured under well controlled conditions in gas and liquid phase. The adaptation for measuring coagulation parameters is achieved by adequate coating of the QCM. QCM measurements of coagulation parameters on a polymer film without NPs has already been demonstrated in previous observations [5,14], but pretreatment with blood plasma of the coatings had to be applied. Following up on this work we here report use of affinity based-NPs adsorbed to polymer thin films for PT measured by the QCM method.

PT tests assess the extrinsic part of the plasmatic coagulation system including factor VII, factor X, V, II and fibrinogen concentration. Anticoagulation by unfractionated heparin leads to prolongation of PT $[15,16]$. As mentioned above, the principle suitability of QCM for PT measurements has been shown $[5,14,17]$. But for the establishment of an exact QCM based method as alternative to standard coagulometer, QCM coatings with significantly enhanced robustness and reusability with no need for pretreatment are still missing. As reference method for our experiments we employed mechanical coagulometry, which can presently still be considered as gold standard. The present study thus mainly focuses on the QCM technique as alternative to that of standard mechanical coagulometry. QCM technique differs from standard coagulometry in the way that it monitors the whole process of coagulation instead of measuring the end point of PT, only. In contrast to optical biosensors, QCM based instruments can also measure viscosity changes in whole blood, mostly obviating the need for centrifugation and thus saving precious time in the point of care setting. The PT of citrated human whole blood with different heparin concentrations was measured with QCM in comparison to coagulometer measurements at the same time under the same conditions. Proof of good correlation with the standard method is of prime importance for the future and further exploitation of the real-time characteristic of QCM sensors for clinical applications. This real-time characteristic can reveal interesting

*Corresponding author: Frank K Gehring, Biosensor Research Group, Institute of Clinical and Experimental Transfusion Medicine, Tuebingen University, Germany, E-mail: frank.gehring@med.uni-tuebingen.de

Received July 31, 2013; Accepted August 21, 2013; Published August 30, 2013

Citation: Hussain M, Sinn S, Zeilinger M, Northoff H, Lieberzeit PA, et al. (2013) Blood Coagulation Thromboplastine Time Measurements on a Nanoparticle Coated Quartz Crystal Microbalance Biosensor in Excellent Agreement with Standard Clinical Methods. J Biosens Bioelectron 4: 139. doi:10.4172/2155-6210.1000139

Copyright: (c) 2013 Hussain M, et al. This is an open-access article distributed under the terms of the Creative Commons Attribution License, which permits unrestricted use, distribution, and reproduction in any medium, provided the original author and source are credited. 
Citation: Hussain M, Sinn S, Zeilinger M, Northoff H, Lieberzeit PA, et al. (2013) Blood Coagulation Thromboplastine Time Measurements on a Nanoparticle Coated Quartz Crystal Microbalance Biosensor in Excellent Agreement with Standard Clinical Methods. J Biosens Bioelectron 4: 139. doi:10.4172/2155-6210.1000139

Page 2 of 6

facets of binding events of bioanalytes and viscosity changes by studying the kinetic of the frequency and the dissipation curves. By applying multiple specially adapted coatings QCM sensors have the capability to determine several different parameters of haemostasis with the same instrument and therefore give an edge over existing clinical standard devices.

\section{Materials and Methods}

\section{Blood sample collection and preparation}

We got the donation of human blood samples approved by the local ethics committee of the university hospital of Tubingen, Germany. Fresh human whole blood from healthy donors should be collected in appropriate syringes containing $1.0 \mathrm{ml}$ citrate solution $(0.106 \mathrm{~mol} / \mathrm{l})$. For our study, collection of samples was performed by the Institute for Clinical and Experimental Transfusion Medicine of the University Hospital of Tubingen, Germany.

\section{QCM sensor setup}

We employed QCM as transducer consisting of a piezoelectric AT-cut-QCM coated with two differently sized gold electrodes $(8 \mathrm{~mm}$ diameter and $5 \mathrm{~mm}$ diameter respectively), one on each side. On such transducers a standing mechanical shear wave within the QCM will be generated on applying alternating voltage to the electrodes. This half wavelength is equal to the thickness of the QCM, why they are also referred to as Thickness Shear Mode Transducers. They are sensitive to mass on ng scale and to physical properties as density, thickness and viscosity of interfacial layers. Thin rigid mass films attached to the upper gold electrode lead to a decrease in resonance frequency proportional to attached mass according to the Sauerbrey equation [18]. The change of frequency and dissipation due to additional effects as viscosity or the density of wetting liquid media is described by the Kanazawa equation [19]. For the description of the frequency and dissipation signals of attached visco-elastic films e.g. cells or bio-layers more complicated equations have to be applied [20-23]. The rheology and the resulting QCM signals of the coagulation process are considered in Müller et al. [14] and Efremov et al. [24]. We used a "qCell T" instrument recently developed by $3 \mathrm{~T}$ analytic, Germany for our real time measurements. This system works with $10 \mathrm{MHz}$ AT-cut QCM sensors with a diameter of $8 \mathrm{~mm}$ and a thickness of $166 \mu \mathrm{m}$. The single channel thermo controlled sensor setup should be constantly kept adjusted to $37^{\circ} \mathrm{C}$ for generating in vivo conditions.

\section{QCM sensor measurements}

QCM containing NPs on polymer layer should be inserted into the qCell $\mathrm{T}$ and Tris buffer ( $\mathrm{pH}$ 7.4) should flow at $37^{\circ} \mathrm{C}$ prior to real time PT measurements. For this purpose we used citrated whole blood mixed with heparin $(0,1.0,1.5,2.0 \mathrm{IU} / \mathrm{ml})$ to achieve different PT ranging from few seconds to tens of seconds. $100 \mu$ of the according blood sample should be incubated at $37^{\circ} \mathrm{C}$ for 1 minute in the incubation chamber of the qCell T. Then, $200 \mu \mathrm{l}$ thromborel, freshly prepared and incubated at $37^{\circ} \mathrm{C}$, should be mixed with whole blood sample. The mixture should immediately be pumped to the measuring chamber at a flow rate of $0.8 \mathrm{ml} / \mathrm{min}$ via running the script control option. The PT with the mechanical coagulometer was performed with equal mixing ratios, volumes and incubation times.

\section{Mechanical coagulometer measurements}

We used a mechanical coagulometer as reference for QCM-PT measurements. This device consists of a thermostat-incubation block at constant $37^{\circ} \mathrm{C}$, to which a $6^{\circ}$ inclined rotating plastic cuvette is mounted. The cuvette holds a stainless steel ball that follows gravity and stays in position on the lower side of the cuvette. The coagulation process is initiated on addition of coagulation stimulant thus increasing the blood viscosity. This ultimately results in higher torque to the steel ball making it go out of position. The rotation of the steel ball is detected by a magnetic field sensor and the PT is determined.

\section{AFM (Atomic force microscopy) measurements}

AFM images of NPs were measured with a VEECO Instruments Nanoscope IVa using contact mode with silicon nitride tip. NPs in absolute ethanol should be spin coated at $2500 \mathrm{rpm}$ on glass slides. The spin coated NP glass slides should be incubated at $38^{\circ} \mathrm{C}$ for 12 hours for drying and immobilization prior to AFM measurements.

\section{Reagents and chemicals}

Poly ethylene (PE), decahydronaphthalene (Decalin), absolute ethanol, ethylene glycol dimethacrylate (EGDMA), di vinyl benzene (DVB), styrene, 1-vinyl-2-pyrrolidone (VP), sulfuric acid $\left(\mathrm{H}_{2} \mathrm{SO}_{4}\right)$ and hydrogen peroxide $\left(\mathrm{H}_{2} \mathrm{O}_{2}\right)$ with highest purity were purchased from Sigma-Aldrich. Thromborel S (purchased from SIEMENS) was reconstituted with $10 \mathrm{ml}$ deionized water and incubated at $37^{\circ} \mathrm{C}$ for 30 minutes. It was stored in refrigerator according to the manufacturer directions and warmed $\left(37^{\circ} \mathrm{C}\right)$ before using it in PT measurements. While sodium chloride (VWR International BVBA) and tris (2-hydroxy ethyl) amine hydrochloride (TRIS) (PAESEL+LORI GMBH \& CO) were used to prepare TRIS buffer $(50 \mathrm{mM}, \mathrm{pH}$ 7.4). Citrate solution $(0.106 \mathrm{~mol} / \mathrm{l})$ was purchased from 9NC S-Monovette, Sarstedt, Nümbrecht-Rommelsdorf, Germany. Heparin-Natrium-25000(Ratiopharm) concentrations were prepared with TRIS buffer ( $\mathrm{pH} 7.4$ ). Piranha solution was prepared by mixing $30 \%$ hydrogen peroxide and concentrated sulphuric acid $(1: 3 \mathrm{v} / \mathrm{v})$.

\section{Equipment}

Mechanical coagulometer Merlin MC 1 (Merlin Medical, ABW Medizin und Technik, Lemgo, Germany) having thermostated incubation block with a constant temperature at $37^{\circ} \mathrm{C}$, qCell $\mathrm{T}(3 \mathrm{~T}$ analytik GmbH, Tuttlingen, Germany), spin-coater (Spin150-v3, Semiconductor Production Systems, Ingolstadt, Germany), atomic force microscope (AFM) (VEECO Instruments Nanoscope IVa, USA), centrifuge (Thermo Scientific, Multifuge 3S-R, Kendro Laboratory Products, Germany).

\section{Experimental procedure}

Poly ethylene NPs synthesis: For poly ethylene NPs synthesis, 500 $\mu \mathrm{l}$ of PE solution in decalin $(1 \% \mathrm{w} / \mathrm{v})$ was pipetted into $10 \mathrm{ml}$ absolute ethanol and kept stirring for 12 hours by using magnetic stirrer. The resulting homogenous solution of NPs was centrifuged at $4000 \mathrm{rpm}$ for 15 minutes and the supernant was discarded. NPs in $500 \mu \mathrm{l}$ absolute ethanol were re-suspended in an Eppendof tube.

QCM sensor cleaning: QCM sensors were cleaned first with acetone followed by deionised water and dried under a stream of nitrogen. For further subsequent cleaning, QCMs were treated with piranha solution for 1 minute, rinsed with deionised water and dried in a stream of nitrogen.

NPs immobilization on QCM: After cleaning the QCMs, the QCM electrode spin coated at $2500 \mathrm{rpm}$ for 20 seconds with $5 \mu \mathrm{l}$ mixture of VP-EGDMA $(1: 2 \mathrm{w} / \mathrm{w})$ or VP-DVB $(1: 2 \mathrm{w} / \mathrm{w})$ or styrene$\operatorname{DVB}(1: 2 \mathrm{w} / \mathrm{w})$. Subsequently, PE-NPs suspensions in $7 \mu$ l ethanol were 
Citation: Hussain M, Sinn S, Zeilinger M, Northoff H, Lieberzeit PA, et al. (2013) Blood Coagulation Thromboplastine Time Measurements on a Nanoparticle Coated Quartz Crystal Microbalance Biosensor in Excellent Agreement with Standard Clinical Methods. J Biosens Bioelectron 4: 139. doi:10.4172/2155-6210.1000139

Page 3 of 6

added on unhardened thin film of monomer: cross-linker $(1: 2 \mathrm{w} / \mathrm{w})$ and spin coated at $2500 \mathrm{rpm}$ for 40 seconds. The spin coated QCMs were incubated at $38^{\circ} \mathrm{C}$ for 12 hours for immobilization and hardening the NPs on polymer prior to real time PT measurements on qCell T.

\section{Results and Discussion}

\section{NPs surface morphology}

Sensor properties as reusability and robustness can be improved by replacing the affinity based polymer thin films by polymer NPs [6]. For this purpose, we chose absolute ethanol for precipitation polymerization, which generated the desired NPs, as can be seen in the AFM image in Figure 1.

PE-NPs surface morphology depicts that individual NPs have an average diameter of around 150-200 nm. Here, we selected three polymer systems namely poly VP cross linked with EGDMA, poly VP cross linked with DVB and poly styrene cross linked with DVB to immobilize the NPs on the QCMs surfaces. The NPs immobilization was achieved by coating the QCM with a $10 \mathrm{~nm}$ thin layer of a prepolymerized monomer-cross linker mixture. Our rationale to use polymer thin film coupled with NPs is to attain the fundamental advantage of polymer affinity towards the protein as well as the increased interacting surface area provided by NPs to improve the sensor quality.

\section{Coagulation measurements}

After NPs characterization by using AFM we applied the QCM sensors to PT measurements by using the sensor platform as mentioned above. Comparing measurements with and without induced coagulation we can clearly distinguish them from one another in perspective of frequency and dissipation shifts and shapes of curve. Exemplary QCM measurements of whole blood samples are shown in Figure 2 .

The PT point in all graphs can be determined as the first local
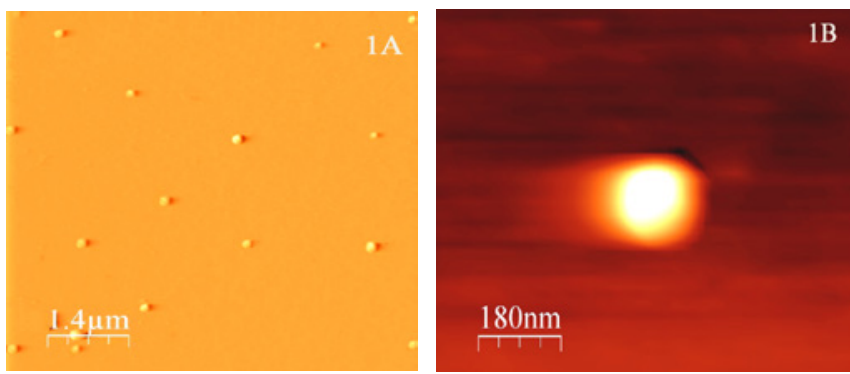

$1 \mathrm{C}$

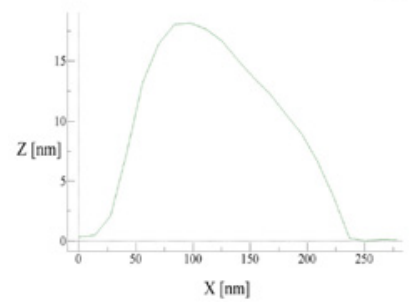

Figure 1: (A) AFM image of poly ethylene NPs in ethanol, Digital Instrument Nanoscope Scan Size $=8 \mu \mathrm{m}$, Scan Angle $=90.0^{\circ}$, Scan Rate $=1.4 \mathrm{~Hz}$, Tip Velocity $=10.0 \mu \mathrm{m} / \mathrm{s}$, Image Data=Height, Data Size=300 nm, (B) 2D image of individual NPs, (C) Graphic representation $X$ and $Z$ axis of $1 B$ image depicting size of NPs.

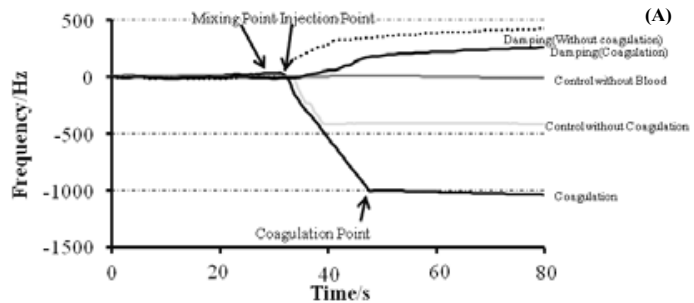

(B)
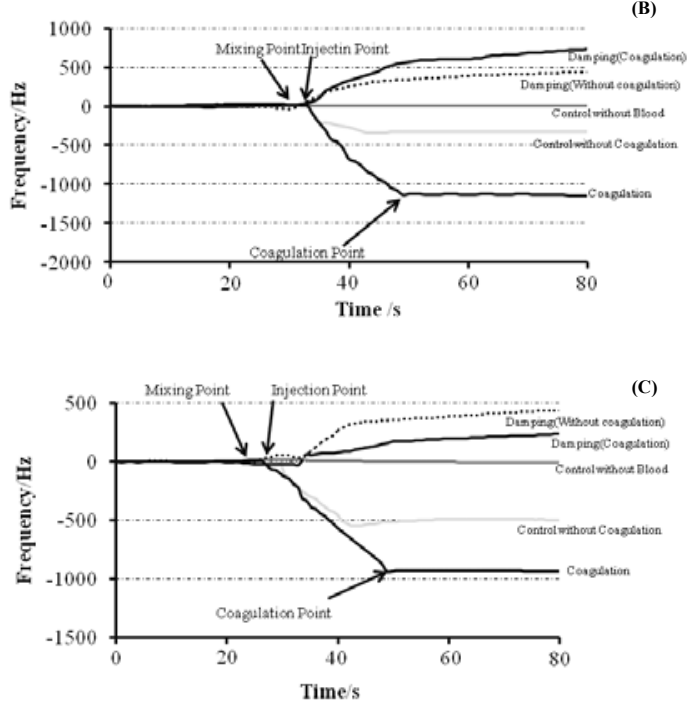

Figure 2: Exemplary measurements of whole blood PT including damping curve as well as two different negative controls: whole blood without blood coagulation and coagulation activator without blood, (A) for NPs immobilized on VP-EGDMA (B) for NPs immobilized on VP-DVB (C) for NPs immobilized on styrene: DVB respectively.

minimum of the characteristic sensor response in terms of frequency shifts and dissipation (damping) curve. The slightly different damping response in the case of $2 \mathrm{~B}$ as compared to $2 \mathrm{~A}$ and $2 \mathrm{C}$ can be attributed to different physico-chemical properties of VP-DVB compared to VPEGDMA and styrene-DVB. In essence, VP-DVB shows lower unspecific adsorption and corresponding damping. For this reason VP-DVB may have a slight advantage over its counterparts, while all three coatings are suitable for measuring PT and deliver comparable results. Standard coagulometric methods record the time span (t) between blood coagulation activation and the point when blood viscosity exceeds a predetermined viscosity threshold, usually termed as the PT. Hence, common coagulometric methods simply display a time value marking a characteristic point of the PT. QCM sensors additionally have the advantage of monitoring the whole kinetic of the coagulation process in real time. The $\mathrm{qCell} \mathrm{T}$ is a thermostated instrument that can monitor every step including dissipation spectrum (half of the half bandwidth of the Lorentz-Curve) in addition to the frequency response (both in Hertz $(\mathrm{Hz})$ ). In order to compare the results obtained from the QCM measurements with those of a standard coagulometer, the end point of the PT has to be determined by picking a significant point of the frequency curve. We concluded that this point is the local minimum where stabilization of the frequency after abrupt shift as depicted in Figure 2 is achieved and defined it as tQCM.

\section{Comparison of coagulation times}

To start with the comparison, Figure 3 demonstrates the PT of human whole blood samples obtained from QCM plotted against PT measured with the coagulometer, respectively. 
Citation: Hussain M, Sinn S, Zeilinger M, Northoff H, Lieberzeit PA, et al. (2013) Blood Coagulation Thromboplastine Time Measurements on a Nanoparticle Coated Quartz Crystal Microbalance Biosensor in Excellent Agreement with Standard Clinical Methods. J Biosens Bioelectron 4: 139. doi:10.4172/2155-6210.1000139

Page 4 of 6
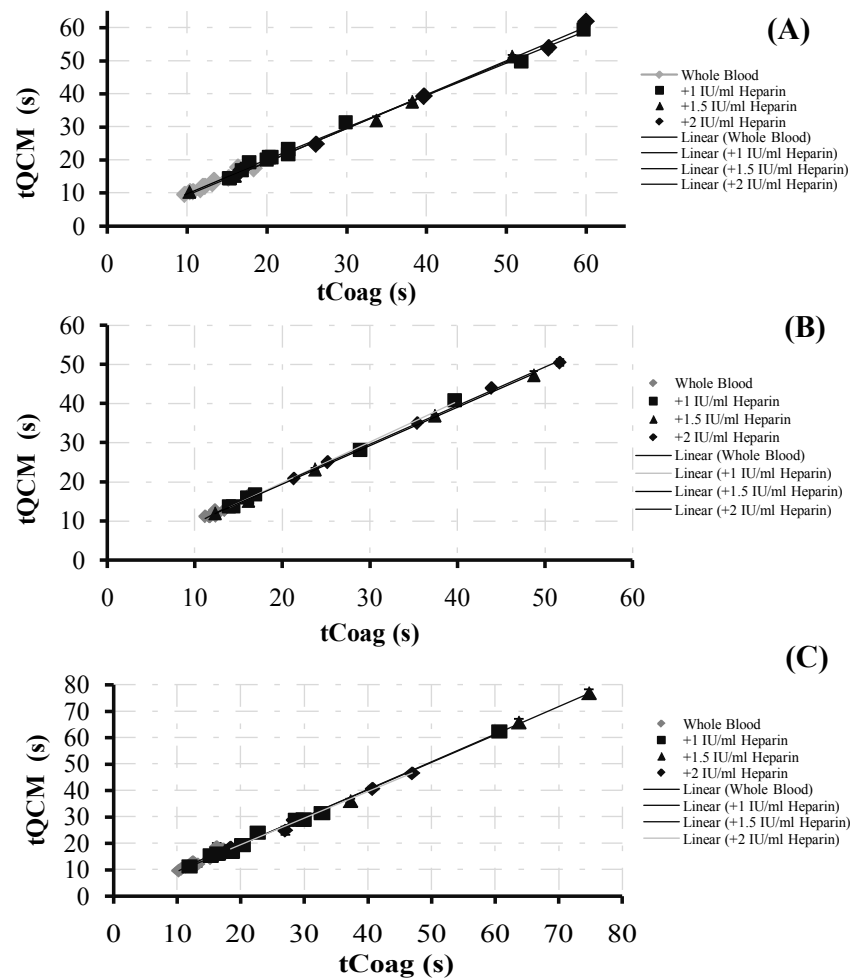

Figure 3: PT of human whole blood obtained from the QCM system plotted against PT gained with a mechanical coagulometer. Whole blood samples and whole blood samples with different heparin concentrations are indicated with appropriate symbols. (A) for NPs immobilized on VP-EGDMA, (B) for NPs immobilized on VP-DVB, (C) for NPs immobilized on styrene-DVB respectively. Each data point represents the mean of two measurements demonstrating error bars \pm SD of two measurements.

The data explains the values for citrated whole blood and blood with the addition of Heparin $(0,1.0,1.5,2.0 \mathrm{IU} / \mathrm{ml})$, respectively. The whole blood measurements with higher amounts of heparin (i.e. 3 or $4 \mathrm{IU} / \mathrm{ml}$ ) resulted in no coagulation in both methods. Ideal correlation between the two methods is represented by the line through origin. The results of all three polymer systems $(3 \mathrm{~A}, 3 \mathrm{~B}, 3 \mathrm{C})$ are within analytical limits of deviation. The Figure depicts that the data lie on the bisecting line representing the excellent correlation with the mechanical coagulometer.

\section{Bland-Altman plot}

For further investigation into the agreement between the two methods we used a Bland-Altman plot [25], as demonstrated in Figure 4.

The Bland-Altman plot demonstrates the agreement between data gained with two different analytical techniques, usually one of which is a newborn or underdeveloped technique. This is plotted by using the difference between the two measurements (i.e., from two different techniques) along $\mathrm{Y}$ axis against the mean along $\mathrm{X}$ axis. As methods with poor agreement can still be in good correlation with each other, the Bland-Altman plot is used to compare the agreement of the two methods rather than correlation. Furthermore the plot also provides a visual overview if the methods compared provide systematically higher or lower values. Our data demonstrates that the three affinity based polymer layers used here are suitable for affinity based NPs adsorption for sensing the PT for human whole blood. As mentioned above the coagulation time, in generally, is defined as time span between addition of coagulation activator and reaching a defined viscosity threshold. That is why different types and brands of mechanical coagulometers are calibrated at similar thresholds for better comparability $[18,26]$. Additionally deviations are also expected due to pre-analytical factors: e.g. methods of whole blood drawing [27], sample preparation and the enzymatic activity of the coagulation reagent used for coagulation $[28,29]$. The data presented here in Bland-Atman plot demonstrates excellent agreement of QCM sensor results with that of standard coagulometers within analytical deviation limits. We reused the sensor layers for up to 10 times with no apparent loss in sensor characteristics.

In general, for PT measurements and beyond, QCM based instruments have great potential in comparison to relevant counterparts. For example, opaque reagents and lipemic blood samples that can interfere with optical coagulation analysers [30] do not interfere with QCM measurements at all. Furthermore QCM based sensors do not require labelling of blood samples to enable specific measurements of e.g. coagulation factors. Surface acoustic sensors (like QCM sensors) are predestinated for measuring visco-mechanical changes and can be regarded as more sophisticated versions of traditional rheometry and thromboelastometry [31,32]. Measuring of visco-elastic properties of the blood clot and changes in mass induced by attachment of e.g. platelets are highly suitable parameters provided by QCM for observation of fibrin polymerization, platelet fibrinogen
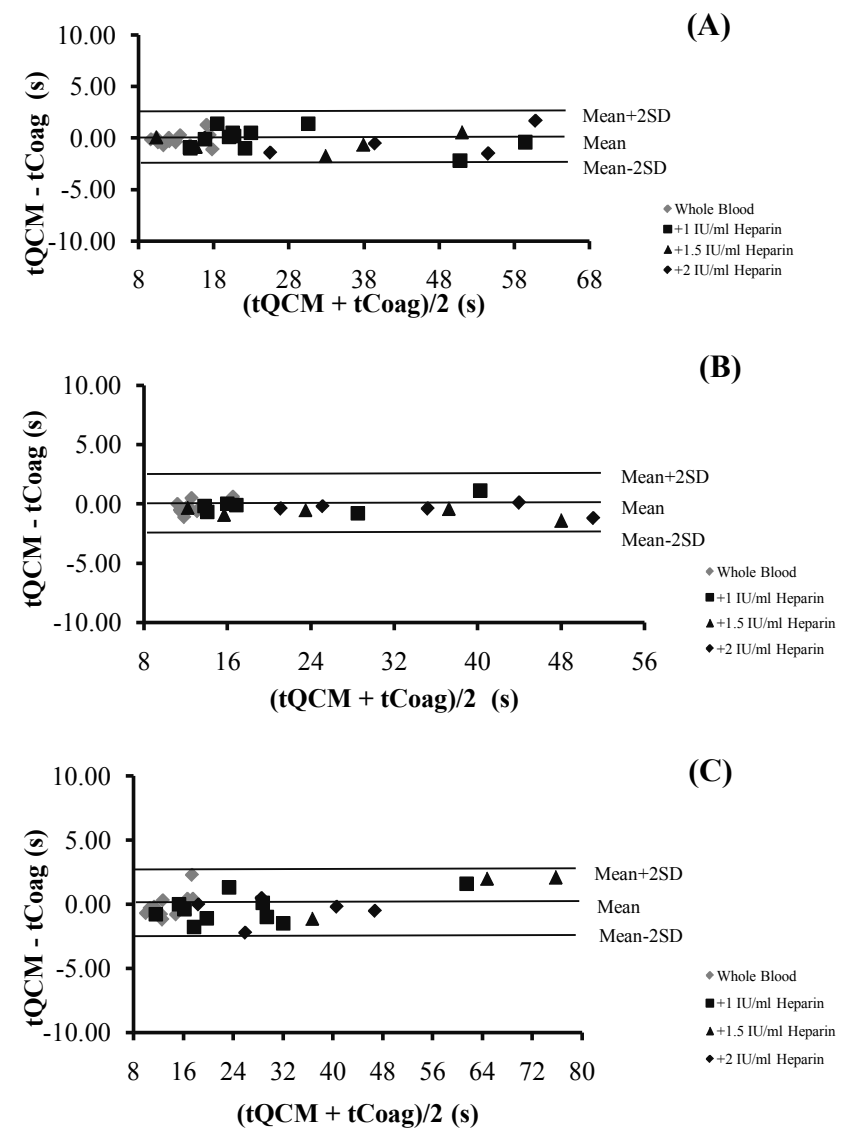

Figure 4: The Bland Altman plot of whole blood PT measurements on QCM and coagulometer. Whole blood samples and whole blood samples with different heparin concentrations are indicated with appropriate symbols, (A) for NPs immobilized on VP-EGDMA, (B) for NPs immobilized on VP-DVB, (C) for NPs immobilized on styrene-DVB respectively. 
Citation: Hussain M, Sinn S, Zeilinger M, Northoff H, Lieberzeit PA, et al. (2013) Blood Coagulation Thromboplastine Time Measurements on a Nanoparticle Coated Quartz Crystal Microbalance Biosensor in Excellent Agreement with Standard Clinical Methods. J Biosens Bioelectron 4: 139. doi:10.4172/2155-6210.1000139

interactions and fibrinolytic processes on non molecular scales. Since QCM measurements can be carried out in whole blood there is no need for prior centrifugation, a factor which saves time and reduces complexity, an important advantage especially in a point of care situation. In respect to integration into fully automated machines for coagulation measurements the excellent potential for miniaturization of QCM is also advantageous.

Regarding the perspective of point of care application of the QCM sensor, it may be of interest that the platform has already proven its potential for platelet aggregation measurements $[5,10]$, determination of fibrinogen concentration (clauss method i.e., [33] ) and automated blood groups analysis [34] in addition to prothrombin time monitoring [14]. The outstanding potential for miniaturization of the QCM sensor offers the possibility of a multi channel measuring platform, in which different haemostatic parameters can be measured simultaneously with automation. Current literature regarding patients undergoing cardio surgical interventions and trauma demonstrates that plasmatic coagulation tests such as prothrombin time (PT), activated partial thromboplastin time (aPTT) or fibrinogen determination (Clauss method) are not sufficient for either thrombosis or bleeding prediction $[9,22,33]$ In such cases devices which can give an overview over different haemostatic parameters are superior to those having specialized test methods. In this application especially thrombo-elastometric methods like ROTEM are currently much valued $[35,36]$. QCM based measurements have the potential to combine the advantages of ROTEM with those of standard coagulometers and thrombo analyzers giving a broad overview over different parameters of haemostasis. Reusability of the QCM sensor layer is important in terms of cost-effectiveness. Additionally, the QCM also offers the possibility to detect coagulation factors e.g. via immobilization of specific antibodies on the sensor surface or via imprinted polymer or NPs coatings [37] Therefore the prospects and potential of the QCM sensor for measuring coagulation parameters are so far not utilized yet. We are convinced that this area deserves the attention of biologists, chemists, physicists and engineers to cope with these challenges due to its multidisciplinary nature.

\section{Conclusions}

In this study we used PE-NPs coupled with VP/styrene polymer system on QCM transducer for recognition of PT of whole blood. Comparative measurements of probes spiked with different concentrations of unfractionated heparin as anticoagulant revealed an excellent correlation of QCM based measurements to standard coagulometer measurements under analytical deviation limits. A QCM sensor contains advantages over other, conventional methods, especially in perspective of point of care application. Moreover the sensor layers have been reused and tested for three months, demonstrating no loss in sensor characteristics.

\section{Acknowledgements}

We acknowledge O. Faul for technical assistance in the laboratory.

\section{References}

1. Zhang L, Gu FX, Chan JM, Wang AZ, Langer RS, et al. (2008) Nanoparticles in medicine: therapeutic applications and developments. Clin Pharmacol Ther 83: 761-769.

2. Ilinskaya AN, Dobrovolskaia MA (2013) Nanoparticles and the blood coagulation system. Part I: benefits of nanotechnology. Nanomedicine (Lond) 8: 773-784.

3. Hussain M, Wackerlig J, Lieberzeit PA (2013) Biomimetic strategies for sensing biological species. Biosensors 3: 89-107.

4. Vaughan O Nature Nanotechnology. doi:10.1038/nnano.2013.1.
5. Sinn S, Müller L, Drechsel H, Wandel M, Northoff H, et al. (2010) Platelet aggregation monitoring with a newly developed quartz crystal microbalance system as an alternative to optical platelet aggregometry. Analyst 135: 29302938.

6. Hussain M, Iqbal N, Lieberzeit PA (2013) Acidic and basic polymers for molecularly imprinted folic acid sensors-QCM studies with thin films and nanoparticles. Sens Act B 21: 176-186.

7. Sniecinski RM, Chandler WL, (2011) Anesth. Analg 113: 1319-1333

8. Hellstern P, Bach J, Simon M, Saggau W (2007) Heparin monitoring during cardiopulmonary bypass surgery using the one-step point-of-care whole blood anti-factor-Xa clotting assay heptest-POC-Hi. J Extra Corpor Technol 39: 8186.

9. Reinhöfer M, Brauer M, Franke U, Barz D, Marx G, et al (2008) The value of rotation thromboelastometry to monitor disturbed perioperative haemostasis and bleeding risk in patients with cardiopulmonary bypass. Blood Coagul Fibrinolysis 19: 212-219.

10. Vikinge TP, Hansson KM, Sandström P, Liedberg B, Lindahl TL, et al. (2000) Comparison of surface plasmon resonance and quartz crystal microbalance in the study of whole blood and plasma coagulation. Biosens Bioelectron 15: 605-613.

11. Bandey HL, Cernosek RW, Lee WE 3rd, Ondrovic LE (2004) Blood rheological characterization using the thickness-shear mode resonator. Biosens Bioelectron 19: 1657-1665

12. Andersson M, Andersson J, Sellborn A, Berglin M, Nilsson B, et al. (2005) Quartz crystal microbalance-with dissipation monitoring (QCM-D) for real time measurements of blood coagulation density and immune complement activation on artificial surfaces. Biosens Bioelectron 21: 79-86.

13. Kotova K, Hussain M, Mustafa M, Lieberzeit PA (2013) MIP sensors on the way to biotech applications: Targeting selectivity.Sens Act B Chem.

14. Müller L, Sinn S, Drechsel H, Ziegler C, Wendel HP, et al. (2010) Investigation of prothrombin time in human whole-blood samples with a quartz crystal biosensor. Anal Chem 82: 658-663.

15. Koestenberger M, Cvirn G, Gallistl S, Muntean W (2004) Drotrecogin alfa activated (recombinant human activated protein $\mathrm{C}$ ) in combination with heparin or melagatran: effects on prothrombin time and activated partial thromboplastin time. Blood Coagul Fibrinolysis 15: 693-697

16. Hunt RL (2011) Evaluation of the prothrombin time, aPTT, and platelet count in the bleeding infant. Neonatal Netw 30: 253-256.

17. Martinez MJ, Inkielewicz-Stepniak I, Medina C, Rahme K, D'Arcy DM, et al. (2012) The use of quartz crystal microbalance with dissipation (QCM-D) for studying nanoparticle-induced platelet aggregation. Int J Nanomedicine 7: 243255.

18. Sauerbrey G, Zeitschrift für Pysik (1959) 155: 206-222.

19. Kanazawa KK, Gordon JG (1985) Anal Chem 57: 1770-1771.

20. Borngräber R, Schröder J, Lucklum R, Hauptmann P (2002) Is an oscillatorbased measurement adequate in a liquid environment? IEEE Trans Ultrason Ferroelectr Freq Control 49: 1254-1259.

21. Lucklum R, Behling C, Hauptmann P (1999) Role of mass accumulation and viscoelastic film properties for the response of acoustic-wave-based chemical sensors. Anal Chem 71: 2488-2496.

22. Voinova MV, Jonson M, Kasemo B (2002) Missing mass effect in biosensor's QCM applications. Biosens Bioelectron 17: 835-841.

23. Gehring FK (2006) Schwingquarzsensorik in Flüssigkeiten: Entwicklung eines Blutanalysegerätes, language: DE, Edn. 1, ISBN-13: 9783865378729, Cuvillier Verlag, International Scientific Publisher, Germany 100.

24. Efremov V, Killard AJ, Byrne B, Lakshmanan RS (2013) The modelling of blood coagulation using the quartz crystal microbalance. J Biomech 46: 437-442.

25. Bland JM, Altman DG (1986) Lancet 1: 307-310.

26. Peng L, Yan C, Wu X, Nie L (2009) Comparability of the results of PT-INR with local MNPT and APTTR with MNAPTT on different coagulation analyzers in China. Int J Lab Hematol 31: 352-358.

27. Peterson P, Gottfried EL (1982) The effects of inaccurate blood sample volume 
Citation: Hussain M, Sinn S, Zeilinger M, Northoff H, Lieberzeit PA, et al. (2013) Blood Coagulation Thromboplastine Time Measurements on a Nanoparticle Coated Quartz Crystal Microbalance Biosensor in Excellent Agreement with Standard Clinical Methods. J Biosens Bioelectron 4: 139. doi:10.4172/2155-6210.1000139

on prothrombin time (PT) and activated partial thromboplastin time (aPTT). Thromb Haemost 47: 101-103.

28. Spannagl M, Moessmer G (2006) Global tests of haemostasis. Hamostaseologie 26: $27-37$.

29. Spannagl M, Dick A, Junker R (2010) POCT in coagulation. Quality assurance. Hamostaseologie 30: 82-90.

30. Arambarri M, Oriol A, Sancho JM, Roncalés FJ, Galán A, et al. (1998) [Interference in blood coagulation tests on lipemic plasma. Correction using n-hexane clearing]. Sangre (Barc) 43: 13-19.

31. Evans PA, Hawkins K, Lawrence M, Barrow MS, Williams PR, et al. (2008) Studies of whole blood coagulation by oscillatory shear, thromboelastography and free oscillation rheometry. Clin Hemorheol Microcirc 38: 267-277.

32. Evans PA, Hawkins K, Lawrence M, Williams RL, Barrow MS, et al. (2008) Rheometry and associated techniques for blood coagulation studies. Med Eng Phys 30: 671-679.
33. Oberfrank S, Drechsel H, Northoff H, Gehring FK (2011) Anwendungen der Schwingquarzsensorik in der Hämostaseologie-Miniaturisierung und Automatisierung. Mikro System Technik-Kongress.

34. Berlitz PA, Claussen J, Döring JS, Drechsel H, Northoff H, Gehring FK (2012) 5th European Conference of the International Federation for Medical and Biological Engineering. Springer Berlin Heidelberg. 1039-1042.

35. Straub A, Schiebold D, Wendel HP, Hamilton C, Wagner T, et al. (2008) Using reagent-supported thromboelastometry (ROTEM) to monitor haemostatic changes in congenital heart surgery employing deep hypothermic circulatory arrest. Eur J Cardiothorac Surg 34: 641-647.

36. Fries D (2011) Coagulation Monitoring using ROTEM $\left({ }^{\circledR}\right)$ : Pro. Anasthesio Intensivmed Notfallmed Schmerzther 46: 312-316.

37. Dixon MC (2008) Quartz Crystal Microbalance with Dissipation Monitoring: Enabling Real-Time Characterization of Biological Materials and Their Interactions. J Biomol Tech 19: 151-158. 